\title{
Space Charge in Nanodielectrics and Its Impact on Electrical Performance
}

\author{
George Chen ${ }^{1,2}$ \\ ${ }^{1}$ The Tony Davies High Voltage Laboratory \\ University of Southampton \\ Southampton, UK \\ gc@ecs.soton.ac.uk
}

\author{
Shengtao $\mathrm{Li}^{2}$ and Lisheng Zhong ${ }^{2}$ \\ ${ }^{2}$ State Key Laboratory of Electrical Insulation for Power \\ Equipment \\ Xi'an Jiaotong University \\ Xian China
}

\begin{abstract}
Nanodielectrics have been actively investigated in last two decades as they have shown some improved dielectric properties that are important for high voltage insulation applications. One of these improvements is the reduction in space charge when the nanodielectrics are subjected to dc electric fields. The formation of deep trap after introducing nanoparticles in the material has been widely reported. However, the mechanisms that are responsible for the charge suppression are not detailed. More importantly, the effect of charge suppression is strongly dependent on the amount of nanoparticles, i.e. loading concentration. In the present paper, a schematic model has been proposed based on deep trap concept. A tunneling process has been introduced when the trapping sites become closer which is the case for high nanofillers concentration. Based on the new model, charge formation and dynamics in nanodielectrics with different loading concentrations can be estimated and electrical performance anticipated. A range of factors that can influence charge trapping/detrapping have been discussed.
\end{abstract}

Keywords-nanodielectrics; space charge; deep traps; tunnelling; electrical perfromance

\section{INTRODUCTION}

Nanodielectrics have been a popular subject within the electrical engineering sector since 1994. Several working group/committee have been formed within CIGRE and IEEE to explore the impact of nanodielectrics. Progress has been made over the last ten years and the improvements in dielectric properties have been widely reported. One of these improvements is that adding nanoparticles into polymeric dielectrics can suppress charge injection [1]. The proposed mechanism was the formation of the deep traps due to the interfacial characteristics between nanoparticles and polymer matrix. Literature search and our own observation reveal that the suppression is only effective at a relative lower loading ratio. Space charge behaviours become more complicated in nanodielectrics with high loading ratios and in many cases the electrical performance deteriorates rather than improves. This degradation in the electrical performance has never been fully discussed.

In the present paper, a simple schematic model has been proposed to explain the observed space charge results for a wide range of nanofillers concentrations. The proposed model is based on the concept of deep traps and charge tunneling between two adjacent nanoparticles in polymer matrix. The new model allows one to estimate the effect of the electric field, trap depth and tunneling distance on charge dynamics in nanodielectrics. The possible implication on the electrical performance of nanodielectrics with a high nanofillers loading ratio is discussed.

\section{SPACE ChARGE IN NANODIELECTRICS}

\section{A. Interface and Its Impact on electrical properties}

It has been generally agreed that the nanoparticles of a material show different properties compared to larger particles of the same material. The interaction between surfaces can appear to be weak on a larger scale but on a nanoscale they are strong. When the particle size in dielectric changes from micrometer to nanometer the most profound effect is the significant increase in surface area to volume ratio as shown in Figure 1.

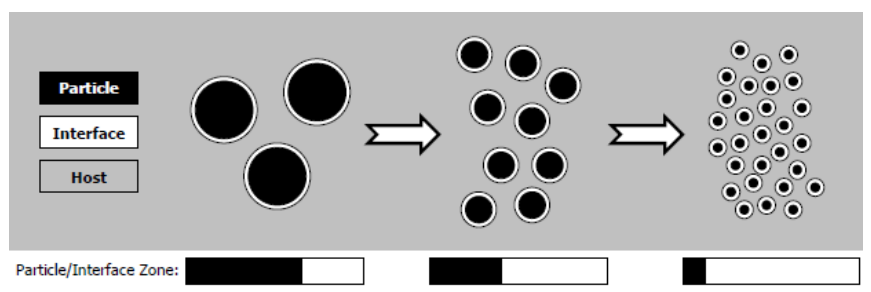

Fig.1 Illustration of how the ratio of the filler material to the interfacial area changes with the size of the fillers [2].

For spherical nanoparticles with a radius of $r$, the surface area, $S$, to volume, $V$, ratio is given by

$$
\frac{S}{V}=\frac{4 \pi r^{2}}{\frac{4}{3} \pi r^{3}}=\frac{3}{r}
$$

It can be seen this ratio will be very larger for nanoparticles. From chemistry point of view, atoms on the surface of a material are often more reactive than those in the bulk. As such it is generally considered that the interface in nanodielectrics plays a key role in determining the electrical performance of nanodieelctrics. A schematic diagram showing the interaction between the nanofiller and polymer matrix is shown in Figure 2. Various models have been proposed with the emphasis on the interface [3]. They provide some physical insight of the interaction zone and assist to understand some of the observed changes. Due to the complexity of the interface, so far no single model has been universally accepted as there is no direct evidence to validate these models. 


\section{B. Deep Traps and Evidences}

The interaction between nanoparticles with polymer matrix is very complicated. One of the widely observed phenomena in nanodielectric is its ability to suppress charge injection. Takada et al. [4] proposed a trapping model based on the concept of 'induced dipole polarization'; it was suggested that the deep potential well induced by nanoparticles becomes trapping sites for carriers, which hinder the movement of carriers and avoid space charge accumulation in the defect positions.

Sufficient experimental evidences are available now to show the reduction of space charge in nanodielectrics [4-5]. TSC measurement has also confirmed the formation of deep trap after introducing nanoparticles as the TSC peak shifts from a lower temperature towards a higher temperature after introducing nanofillers [6].

\section{Effect of Nanoparticle Concentration}

It is worth to mentioning that the suppression effect of space charge is only effective when in low concentration. This fact has rarely been reported in literature. Our experience has shown when in higher concentration space charge dynamics become more complicated. Two different charge profiles have been observed i.e. (i) complicated charge profiles [7] and (ii) less space charge [8].

\section{SCHEMATIC MODEL}

The concept of deep trap introduced by the nanoparticles has been widely accepted. However, the exact mechanism has not been fully explained, especially in the case of space charge measurements. The observed space charge suppression is typically related to nanodielectrics with a lower loading concentration. More complicated charge distributions are often observed in nanodielectrics with a high loading concentration. Based on the deep trap concept, we intend to explain the two phenomena by proposing a schematic model as shown in Figure 2.

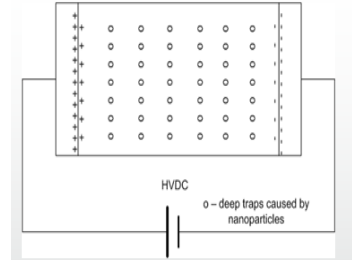

(a) low concentration

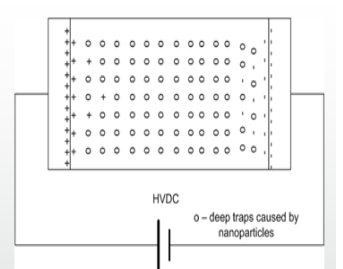

(b) high concentration
Fig. 2 Schematic model showing possible charge distribution in nanodielectric with low and high loading concentrations.

We believe the charge injection still takes place when the electric field exceeds the threshold value. The injected charge will be captured by deep traps introduced by nanoparticles. Initially, the traps in the region of sub-surface will be filled up. The effect of the trapped charge will produce a field that is opposite to the applied field, resulting in a reduction of the electric field at the interface. This will hinder further charge injection. When in lower concentration, the distance between these deep traps is very large. The captured charges may not have sufficient energy to escape the deep traps, therefore, the charge movement to the adjacent traps is difficult. The situation becomes stable. On the other hand, all the direct space charge measurement techniques have a limited spatial resolution it is not possible to separate the capacitive/induced charges on the electrode and trapped charge very close to the electrode. So the observed result is typically considered as the capacitive charge on the electrode, leading to a conclusion of the absence of space charge in nanodielectrics.

Space charge in nanodielectrics with a high loading concentration has not been fully explored. There are reports in literature, showing more complicated space charge patterns in nanodieelctrics with a loading concentration higher than $5 \%$ [7]. We believe that the tunneling process may be responsible for the observed phenomenon. When in a higher concentration, the injection and capture processes described above still apply. However, the distance between two adjacent traps becomes shorter. Instead of overcoming the energy barrier, the tunneling process can take over for the movement of charges. The initial captured charge carriers now can move to the traps adjacent and the process continues. Consequently, the traps near to the sub-surface now are empty and the electric field at the interface increases, resulting in further charge injection. As the injected charges move into the bulk of the sample, their presence can be detected.

For the tunneling between the two conducting particles the conductance is described as [9]:

$$
g_{i, j}=g_{0} \exp \left(-\frac{2 d}{d_{0}}\right)
$$

where $g_{0}$ is a constant, $d$ is the separation distance between two adjacent particles in the field direction, $d_{0}$ is the characteristic tunneling distance. In the case of spherical shaped nanoparticles with a same diameter, assuming the nanoparticles are uniformly distributed in polymer matrix, the separation distance between the two adjacent nanopartilces is given by

$$
d=r\left\{\left[\frac{4 \pi}{3}\left(1+\frac{1-F}{F} \frac{\rho_{f}}{\rho_{m}}\right)\right]^{1 / 3}-2\right\}
$$

where $r$ is the radius of nanoparticle, $F$ is the weight fraction of nanoparticles, $\rho_{\mathrm{f}}$ and $\rho_{\mathrm{m}}$ are the density of nanoparticles and polymer matrix respectively.

For a typical dielectric, the tunneling current is affected by the energy of the deep trap and the modification by the electric field as shown in equation below.

$$
J_{F N}=\frac{A E^{2}}{E_{t}} \exp \left(-\frac{B E_{t}}{E}\right)
$$

where $A$ and $B$ are constants, $E$ the electric field and $E_{t}$ the barrier height or trap depth. For a nanodileectric where deep traps have been introduced, one should combine the two equations to estimate the charge transport characteristics, i.e.

$$
J=\frac{C E^{2}}{E_{t}} \exp \left(-\frac{B E_{t}}{E}\right) \exp \left(-\frac{2 d}{d_{0}}\right)
$$

where $C=A \times g_{0}$ is a new combined constant.

Based on the above equation, there are three parameters that can influence charge transport characteristics in a nanodielectric and they are the separation distance between two adjacent nanoparticles, trap depth and the electric field. 
There are ranges of factors that can influence the separation distance and trap depth. Additionally, there are other factors that may affect charge injection and generation in nanodielectrics. All these will be briefly discussed in the following sections.

\section{A. Effect of trap depth}

The space charge suppression is established on the concept of introducing deep traps following the interaction between nanofillers and polymer matrix. Any factor that can affect the interaction will influence the trap depth. Nature of particles, polymer matrix, surface treatment and moisture are the major factors that have impact on the depth of traps which will contribute towards the observed charge dynamics in the nanodielectrics.

\section{B. Effect of seperation distance}

The separation distance between nanoparticles plays a significant role in determining charge transport processes. The major factors that influence the separation distance are concentration, particles size/shape and distribution/dispersion of nanoparticles. For the same concentration, the smaller the diameter of the nanofillers, the shorter the deparation distance between the two adjacent nanofillers.

The dispersion/distribution of nanofillers in polymer matrix is also important as shown in Figure 3 where a few some possible dispersion/distribution of nanofillers are iluustrated. The tunneling distance changes drasticaly for different dispersion/distribution.

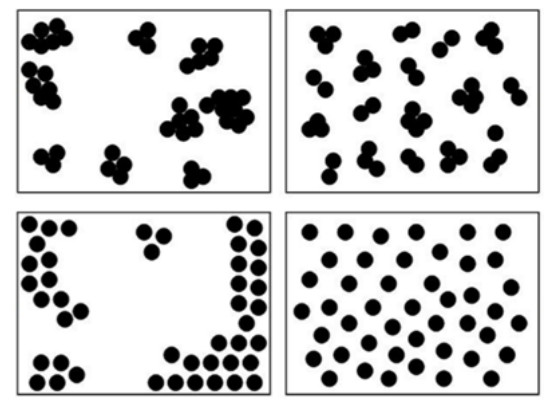

Fig.3 Illustration of the possible dispersion and distribution of nanoparticles inside a polymer nanocomposites [10].

\section{Effect of electric field}

The effect of electric field has two folds. On one hand it increases the charge injection into the nanodielectric when the higher electric is applied. On the other it enhances the charge tunneling as described in equation (5). When the applied field is high enough it is still possible for the injected charge moves into the bulk even for nanodielectrics with a low nanofillers concentration. Therefore, it is important to examine space charge characteristics in the high field region for nanodielectrics.

\section{Effect of moisture}

The effect of moisture also has two folds. Most of nanofillers are prone to moisture due to their reactive surfaces. The direct impact of the absorbed moisture is to modify the surface characteristics and alters trap depth. This will change charge dynamics in the nanodielectric. Secondly, water can easily be ionized when subjected to high electric fields, producing ionic charges which will have very different characteristics compared with the injected charges. The combination of the two makes charge dynamics more complicated.

\section{E. Effect of temperature}

Although the temperature is not featured in equation (5), its impact on charge dynamics is obvious. Firstly, it promotes charge injection. In addition to deep traps in the nanodielectric there are also shallow traps. Higher temperature will enhance charge move from these traps.

\section{F. Effect of crystallinity}

Some of polymeric materials are semicrystalline in nature. Nanoparticles are most likely present in amorphous region. This means that clusters can be formed, deviating from uniform dispersion of the nanofillers.

\section{G. Implication for high concentration}

From equation (5) it becomes apparent that the conduction current increases significantly when the nanodielectric with a high loading concentration is exposed to high electric fields. This will lead to a deteriorated performance in terms of electrical breakdown of the nanodieelctric. In addition, due to higher conduction current, it is unlikely that space charge is able to accumulate in the nanodielectric. The high conduction current and lower breakdown strength may limit its applications under HVDC conditions. DC breakdown strength may not necessarily be improved for nanodielectrics even with a low nanofiller loading as the breakdown typically means that the sample is subjected to very high fields.

\section{SuPPORTING EVIDENCES}

There are many factors that can influence the charge dynamics in nanodielectrics. Sufficient evidences in the literature provide a strong support for the proposed model. A few examples have been selected with an emphasis on DC characteristics to demonstrate the application of the model.

\section{A. Conduction process in nanodielectric}

According to the model proposed the conduction current in a nanodielectric is expected to change with concentration. At lower concentration the conductivity should decreases due to the introduction of deep traps. However, when in high loading concentration, one expects to see an increase in the conductivity. This has been observed in our previous research [11] as shown in Figure 4.

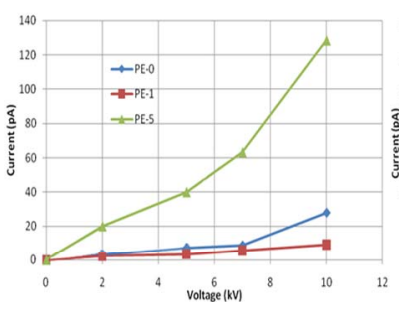

(a) $\mathrm{T}=20^{\circ} \mathrm{C}$

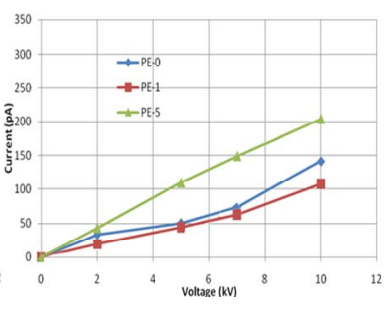

(b) $\mathrm{T}=60^{\circ} \mathrm{C}$
Fig. 4 Conduction current versus the applied voltage for nanoalumina filled LLDPE at (a) $20^{\circ} \mathrm{C}$ and (b) $60^{\circ} \mathrm{C}$ [11]. 
Compared to the LLDPE sample without nanoparticles, it can be seen that the conduction current in LLDPE sample loaded with $1 \mathrm{wt} \%$ alumina nanoparticles decreases for the same applied voltage. However, when the alumina nanoparticles loading increases to $5 \mathrm{wt} \%$ the conduction current becomes higher. The above observation still holds when the measurement temperature increases to $60^{\circ} \mathrm{C}$.

\section{B. Space charge dynamics}

Figure 5 shows the effect of surface treatment and applied electric field on space charge formation in 5\% nanosilica loaded polyethylene. When in slight high concentration, it is possible to observe the injected charge as shown in (b). It can clearly be seen that the effect of surface treatment when comparing (b) and (c). Generally, the surface treatment is to improve nanofillers dispersion. In the example shown here the surface treatment may not only improve nanofillers dispersion but also increase trap depth. Consequently, charge suppression is more effective.

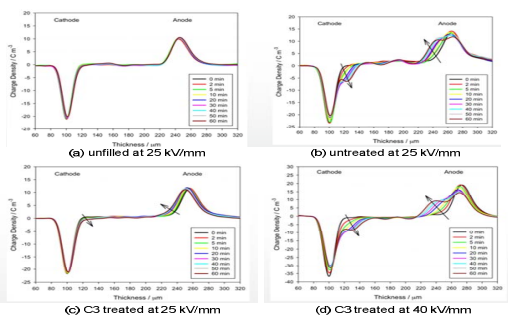

Fig. 5 Space charge in $5 \mathrm{wt} \%$ nanosilica loaded polyethylene [12].

When comparing (c) and (d), it is apparent that the high applied field can move the injected charge into the bulk of the nanodielectric as discussed in the earlier section. It can be seen there is little injection at lower applied field $(25 \mathrm{kV} / \mathrm{mm})$ but significant at higher applied field $(40 \mathrm{kV} / \mathrm{mm})$.

\section{DC breakdown strength}

Charge dynamics, including charge formation and transport, play a significant role in DC breakdown process. Based on the proposed model for nanodielectrics, the charge suppression is only effective when in low loading ratio and at low electric field, it is unlikely to see any improvement in DC breakdown strength. At high fields the presence of traps may promote charge tunneling, one may expect to observe the deteriorated performance as shown in Table II.

TABLE II WEIBULL PARAMETERS FOR DC BREAKDOWN OF NANOSILICA BASED POLYETHYLENE

\begin{tabular}{|c|c|c|}
\hline Sample & Breakdown strength (kV/mm) & Slope \\
\hline Unfilled & 378.8 & 6.4 \\
\hline 2wt\% untreated & 332.6 & 5.2 \\
\hline 2wt\% C3 treated & 292.0 & 10.2 \\
\hline $5 \mathrm{wt} \%$ untreated & 283.8 & 8.9 \\
\hline $5 \mathrm{wt} \%$ C3 treated & 238.5 & 6.6 \\
\hline $10 \mathrm{wt} \%$ untreated & 197.1 & 3.1 \\
\hline $10 \mathrm{wt} \% \mathrm{C} 3$ treated & 199.3 & 8.8 \\
\hline
\end{tabular}

A general decline in the breakdown strength has been observed with the increasing loading ratio. It is noticed that the difference in the breakdown strength exists for untreated and treated samples and a slight higher value is obtained for untreated samples. The possible reason is the uniform dispersion/distribution may promote charge tunneling in terms of a shorter tunneling distance compared to the cluster dispersion/distribution (see Figure 3).

\section{CONCLUSIONS}

A schematic model has been proposed to explain charge dynamics in nanodielectrics. It is based on the concept of deep traps introduced due to the interaction between nanofillers and polymer matrix. Tunneling mechanism has been applied to explain nanodielectrics with a high nanofiller concentration.

A mathematical expression has been proposed to characterize the tunneling conduction which contains three major parameters, the electric field, trap depth and tunneling distance. Factors that may affect trap depth and tunneling distance together with the other factors that influence charge dynamics have been identified.

There are overwhelming evidences in the literature in support of the proposed model. Based on the model, care has to be taken when exploring nanodielectrics for HVDC applications. DC performance of nanodielectrics at high fields is likely to deteriorate, especially for nanodielectrics with a high nanofiller ratio.

\section{REFERENCES}

[1] T. Takada, Y. Hayase and Y. Tanaka, "Space charge trapping in electrical potential well caused by permanent and induced dipoles for LDPE/MgO nanocomposite", IEEE Trans. Dielectr. Electr. Insul, Vol.15, p 152-160, (2008).

[2] T. Andritsch, "Epoxy based nanodielectrics for high voltage DC applications - synthesis, dielectric properties and space charge dynamics," Ph.D. Thesis, Delft University of Technology, (2010).

[3] S. T. Li, G. L. Yin, G. Chen, J. Y. Li, S. N. Bai, L. S. Zhong, Y. X. Zhang and Q. Q. Lei, "Short-term breakdown and long-term failure in nanodielectrics: A Review”, IEEE, Trans. Dielectr. Electr. Insul, Vol. 17, p 1523-1535, (2010)

[4] T. Takada, Y. Hayase, Y. Tanaka and T. Okamoto, "Space charge trapping in electrical potential well caused by permanent and induced dipoles for LDPE/MgO nano - composite". IEEE, Trans. Dielectr. Electr. Insul, Vol.15, p 152-160 (2008).

[5] S. Ju, M. Chen, H. Zhang and Z. Zhang, "Dielectric properties of nanosilica/low-density polyethylene composites: The surface chemistry of nanoparticles and deep traps induced by nanoparticles", eXPRESS Poly. Lett, Vol. 8, 682-691, (2014).

[6] B. Han, X. Wang, Z. Sun, J. M. Yang, and Q. Q. Lei, "Space charge suppression induced by deep traps in polyethylene/zeolite nanocomposite", Appl. Phys. Lett., Vol. 102, pp. (012902) 1-4, (2013).

[7] G. Chen, C. Zhang and G.C Stevens, "Space charge in LLDPE loaded with nanoparticles" CEIDP, Vancouver, Canada, pp. 275 -278, (2007)

[8] Y. Wang, G. Chen and A. S. Vaughan, "Space charge dynamics in silica-based polyethylene nanocomposites". CEIDP, Des Moines, (2014)

[9] G. Ambrosetti, C. Grimaldi, I. Balberg, T. Maeder, A. Danani, and P. Ryser, "Solution of the tunnelling-percolation problem in the nanocomposite regime", Phys. Rev.B 81, 155434 (2010).

[10] G. Chen, J. T. Sadipe, Y. Zhuang, C. Zhang and G. C. Stevens, "Conduction in liner low density polyethylene nanocomposite", IEEE ICPADM, pp. 845 - 848, Harbin, China, (2009).

[11] T. Andritsch, R. Kochetov, P. H. F. Morshuis and J. J. Smit "Influence of manufacturing on dielectric performance of nanocomposites" 2013 IEEE ICSD, Bologna, Italy, June 30 - July 4, (2013)

[12] Y. K. Lau, A. S. Vaughan, G. Chen, I. L. Hosier, A. F. Holt and K Y. Ching, "On the space charge and DC breakdown behavior of polyethylene/silica nanocomposites". IEEE, Trans. Dielectr. Electr. Insul, Vol. 21, p 340-351, (2013). 